SILVA, M.A.M.; COUTINHO, K.D.; WANDERLEY, C.D.V.; GUERRA, P.V.A.; PAIVA, A.K.O.; GUERRA NETO, C.L.B. (2012).

\title{
LIMPEZA A PLASMA PARA MELHORAR A ADESÃO SUPERFICIAL DE FILMES
} DE ÓXIDOS DE TITÂNIO

Marco Aurélio Medeiros da Silva.

Programa de pós-graduação em ciências da Saúde - PPgCSA - Natal/ RN - Brasil

marcoaureliobucofacial@yahoo.com.br

Karilany Dantas Coutinho

Universidade Federal do Rio Grande do Norte, Departamento de Engenharia Biomédica, Campus Universitário, Lagoa Nova, Natal, RN, Brasil. CEP: 59072-970. karilany@ufrnet.br

Caroline Dantas Vilar Wanderley

Universidade Federal do Rio Grande do Norte, Departamento de Engenharia Biomédica,

Campus Universitário, Lagoa Nova, Natal, RN, Brasil. CEP: 59072-970.

carolinevilar@gmail.com

Paulo Victor de Azevedo Guerra

Universidade Federal do Rio Grande do Norte, Escola de Ciência e Tecnologia, Campus

Universitário, Lagoa Nova, Natal, RN, Brasil. CEP: 59072-970. paulo_victor_7@hotmail.com

Ana Karenina de Oliveira Paiva

Universidade Federal do Rio Grande do Norte, Escola de Ciência e Tecnologia, Campus Universitário, Lagoa Nova, Natal, RN, Brasil. CEP: 59072-970. kareninapaiva@outlook.com

\section{Custódio Leopoldino Brito Guerra Neto}

Universidade Federal do Rio Grande do Norte, Departamento de Engenharia Biomédica,

Campus Universitário, Lagoa Nova, Natal, RN, Brasil. CEP: 59072-970.

custodioguerra@yahoo.com.br

\section{RESUMO}

O tratamento para modificação superficial requer um substrato com alto grau de limpeza, a fim de promover um aumento da energia superficial e melhorar a adesão da camada formada ao substrato. Técnicas que estão em crescente uso possuem geralmente o plasma como fonte energética. Nesse trabalho, utilizou-se o plasma produzido em descarga por cátodo oco, com diferentes atmosferas, a fim de estudar a sua influência na despassivação da superfície de titânio. Foram utilizadas 06 placas de Ti cp (grau II), de tamanho 10 x 10 x $1 \mathrm{~mm}$. Estas amostras foram divididas em três grupos para limpeza a plasma em catodo oco com atmosfera de $\mathrm{Ar}, \mathrm{H}_{2}$ e $\mathrm{Ar} / \mathrm{H}_{2}(50-50 \%)$ a pressão em torno de 0,7 mbar e temperatura de $200^{\circ} \mathrm{C}$ nos tempos de 20 e 60 minutos e seguido uma condição de oxidação a plasma a $500^{\circ} \mathrm{C}$ por 1 hora, com teor $10 \%$ de $\mathrm{O}_{2}$ numa pressão de 2,2 mbar. Após oxidação as amostras foram submetidas à avaliação através de análise de ângulo de contato pela técnica da gota séssil (molhabilidade), análise por difração de raios-X (DRX) e rugosidade.

PALAVRAS-CHAVE: Limpeza a plasma, implante dental, Oxidação, Descontaminação de Ti. 


\title{
PLASMA CLEANING TO IMPROVE SURFACE ADHERENCE OF TITANIUM OXIDE FILMS
}

\begin{abstract}
Surface modification requires a very clean substrate to increase surface energy and improve adherence of the layer formed on the substrate. Techniques with plasma as the energy source are increasingly being employed. This study used plasma produced in hollow cathode discharge in different atmospheres in order to study its influence on depassivation of the titanium surface. Six $10 \times 10 \times 1 \mathrm{~mm}$. Ti plates (grade II) were used. These samples were divided into three groups for plasma cleaning in hollow cathode in an atmosphere of Air, $\mathrm{H}_{2}$ and $\mathrm{Air} / \mathrm{H}_{2}(50-50 \%)$ at a pressure of around $0.7 \mathrm{mbar}$ and temperature of $200^{\circ} \mathrm{C}$ for 20 and 60 minutes, followed by plasma oxidation at $500^{\circ} \mathrm{C}$ for 1 hour, with $10 \% \mathrm{O}_{2}$ content at a pressure of 2.2 mbar. After oxidation, the samples were assessed by analyzing the contact angle using the sessile drop technique (wettability), x-ray diffraction (XRD) analysis and surface roughness.
\end{abstract}

KEYWORDS: Plasma cleaning, dental implant, Oxidation, Ti decontamination.

\section{LIMPEZA A PLASMA PARA MELHORAR A ADESÃO SUPERFICIAL DE FILMES DE ÓXIDOS DE TITÂNIO}

\section{INTRODUÇÃO}

Nos últimos anos tem crescido o uso da tecnologia a plasma para modificação de superfícies, sendo esta técnica já bastante utilizada na área biomédica em tratamento de superfícies do titânio (Ti). Existem também muitos trabalhos de oxidação, usando plasma como fonte energética. Nesses trabalhos a grande preocupação é voltada, principalmente, para aspectos micro-topográficos e de textura, visando o aumento da molhabilidade. Em odontologia estes materiais têm sido pesquisados com o intuito de tornar a deposição óssea em suas superfícies mais eficiente. Hoje o material mais utilizado para a confecção de implantes odontológicos é o titânio e suas ligas (Ti6Al4V), devido à sua alta biocompatibilidade e resistência à corrosão. Na Engenharia Tecidual há um crescente interesse em se desenvolver uma nova geração de materiais que, além de biocompatíveis e resistentes à corrosão, tenham boa molhabilidade para garantir uma maior integridade da interface osso/biomaterial. (ALBREKTSSON, T.\& SENNERBY, 1990). No caso de implantes dentais, após a fabricação, os mesmos são submetidos a uma série de procedimentos que incluem protocolos de limpeza e passivação. Esses procedimentos são recomendados por órgãos que estabelecem normas e condutas técnicas para fabricação de materiais para uso biológico, com a finalidade de obter superfícies que possam ter melhor interação com os tecidos, onde são implantados (ATMS, 1990). As alterações sofridas pelos implantes durante o processo de usinagem podem incluir contaminantes orgânicos e inorgânicos, provenientes dos processos de fabricação. Em seguida, os implantes são submetidos aos processos de limpeza que podem ser através de métodos mecânicos, físicos e químicos. Os métodos mecânicos são realizados as custas de jateamento sobre o substrato com partículas de diversas granulações. Dentre os métodos físicos podemos 
citar: laser, spray térmico, plasma spray, PVD, implantação iônica, implantação de íons e deposição e tratamento com descarga incandescente de plasma, já o processo químico é realizado à custa de banhos sucessivos de ácidos, tratamento alcalino, peróxido de hidrogênio, sol-gel e oxidação anódica. (X. LIU, C.PAUL K., D., CHUANXIAN 2005) O método químico é o mais utilizado, limpa e trata a superfície, porém poderá levar à incorporação de elementos nocivos ao material e tornar necessário etapas adicionais para descontaminação, aumentando tempo e custo a esse processo, além de causar problemas no descarte de resíduos ao meio ambiente, após o seu uso, ocasionando danos de difícil reparação para o mesmo. (CHANG H. Y., et al., 2004)

O plasma tem sido bem estabelecido na limpeza e processamento de superfície na indústria de microeletrônicos (MITTAL K.L., 1979) e tem atraído á atenção na área de biomateriais (GOMBOTZ W.R., HOFFMAN A.S.,CRC 1987). Embora esse método tenha sido usado vastamente em pesquisa de biomateriais, as informações são limitada sobre os parâmetros do processo.

Uma das grandes vantagens desse método está na versatilidade da energia do plasma. Num mesmo equipamento é possível limpar, modificar uma superfície e esterilizar-la. Esse processo consiste em oxidar a peça numa atmosfera altamente ionizada e com a finalidade de tornar o ambiente mais hostil é utilizada a configuração cátodo oco. A alta ionização é conseguida porque os elétrons do plasma são obrigados a refletirem sucessivamente entre duas superfícies catódicas, onde os elétrons são repelidos pelo cátodo central que pode ser a peça que se deseja oxidar em direção aos cátodos externos. Ao se aproximarem destes, são também repelidos, realizando assim, um movimento de zig-zag que aumentará a taxa de ionização da região em questão. Uma alta densidade de íons significará maior bombardeamento na superfície, ocasionando maior incidência energética. Esta configuração de eletrodos é utilizada quando se deseja aumentar o dano superficial, ou seja, para uma mesma temperatura de processo, produz-se mais defeitos na superfície que na oxidação com a descarga planar. (ALVES JR. C, 1995)

Alguns autores têm investigado o tratamento em descarga planar para limpeza de superfície e modificação de biomateriais metálicos de titânio. Em geral foi utilizada como atmosfera, Ar e $\mathrm{O}_{2}$, onde o primeiro serviu para remoção de camadas de superfície nativas, compostos de óxido e impureza de tratamento prévios de superfície e o segundo para sua reoxidação (X. LIU, C.PAUL K., D., 2005). Foi reportado também que um mistura gasosa de plasma $\mathrm{H}_{2}$ / Ar, pode ser eficiente na remoção de óxidos metálicos durante a limpeza, onde radicais de hidrogênio pode ajudar a reduzir óxidos, enquanto que o argônio atua aumentando a eficiência da taxa de ionização. (HSIEH J.H., LI C., 2006)

$\mathrm{O}$ presente estudo tem por objetivo avaliar o efeito de $\mathrm{Ar}$ e $\mathrm{H}_{2}$, no processo de limpeza prévia à oxidação de superfícies em titânio, usando o método de descarga em cátodo oco.

\section{MATERIAIS E MÉTODOS}

Neste trabalho foram utilizadas 06 de placas cp de Ti (grau II), medindo $10 \mathrm{~mm}$ x $10 \mathrm{~mm}$ x $1 \mathrm{~mm}$ (largura x altura x espessura), que foram divididas em três grupos prévios de limpeza (Ar, $\mathrm{H}_{2}$ e Ar/ $\mathrm{H}_{2}$ ), seguido de oxidação em plasma, (Tabela 1). Após as oxidações das 06 placas em descarga de catodo oco (HCD), todas foram submetidas a ensaios de molhabilidade, análise de difração de raios-X e microdureza. 
Tabela 1 - Condições de tratamento, gás utilizado, tempo e porcentagem de $\mathrm{O}_{2}$.

\begin{tabular}{l|l|l} 
Condições de limpeza & Tempo (Min) & Atmosfera de Oxigênio \\
\hline Argônio & 20 & $10 \%$ \\
Argônio & 60 & $10 \%$ \\
Hidrogênio & 20 & $10 \%$ \\
Hidrogênio & 60 & $10 \%$ \\
Argônio + Hidrogênio & 20 & $10 \%$ \\
Argônio + Hidrogênio & 60 & $10 \%$
\end{tabular}

\subsection{APARATO EXPERIMENTAL}

O aparato experimental utilizado para a oxidação em plasma consiste de uma câmara hermeticamente fechada contendo um ânodo na parte superior e uma base catodicamente polarizada (figura 1). Sobre essa base foi posicionada a peça a ser oxidada. Em torno dessa peça foi colocada a configuração em cátodo oco, consistindo de um anel nas laterais da mesma, com uma tampa na parte superior (figura 2). Todas as partes metálicas foram confeccionadas de aço inoxidável, sendo as paredes internas do cátodo oco revestidas com $\mathrm{Ti}$, para evitar contaminação da peça. Fixou-se a distância entre a superfície da amostra e a tampa superior, aqui denominada distância entre cátodos $\left(\mathrm{d}_{\mathrm{c}}\right)$, em $9 \mathrm{~mm}$.

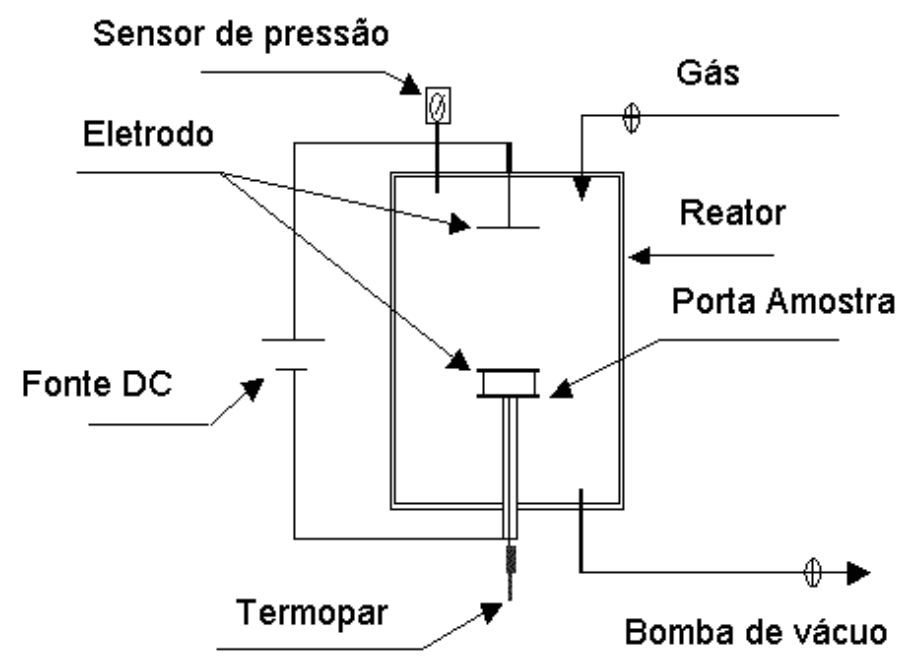

Figura1 - Diagrama esquemático do reator a plasma e periféricos. 


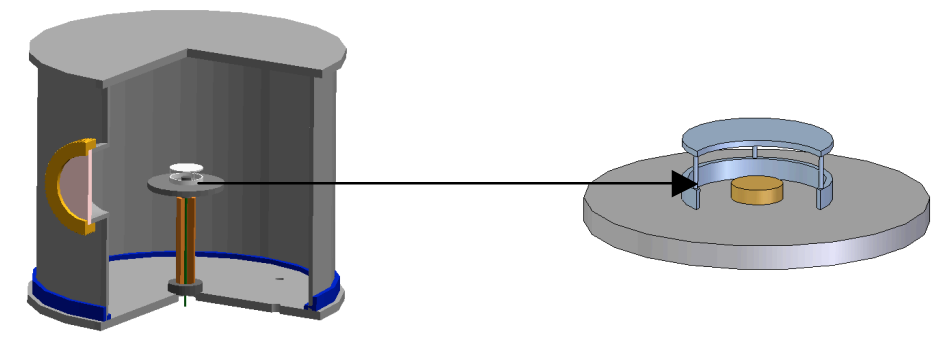

Figura 2 - Vista interna do reator apresentando detalhes da configuração cátodo oco.

\subsection{LIMPEZA E TRATAMENTO DAS AMOSTRAS}

Inicialmente as placas foram tratadas previamente com argônio e hidrogênio e uma combinação destes, na proporção de 1:1, em uma pressão em torno de 0,7 mbar e temperatura de $200^{\circ} \mathrm{C}$ e na seqüência uma oxidação de $\mathrm{O}_{2}$ de $10 \%$, numa pressão 2,2 mbar a $500^{\circ} \mathrm{C}$ por 1 hora respectivamente para cada condição de limpeza. A temperatura foi medida por um termopar cromel-alumel em contato com porta-amostra e controlada pela variação contínua da tensão e da corrente entre os eletrodos. Depois de terminado o tratamento da superfície, todas as amostras foram submetidas a um rígido protocolo de limpeza para remoção de gorduras, proteínas e carboidratos feitos com uma solução de DEIV 3E em ultra-som, durante 10 minutos. Lavagem em álcool absoluto, sendo submetido por 10 minutos em ultra-som. Outra lavagem, agora em água destilada, permanecendo por 10 minutos em ultra-som. Cada etapa foi repetida duas vezes. As amostras foram secadas com secador por um minuto e acondicionadas em embalagens apropriadas para esterilização com peróxido de hidrogênio.

\subsection{CARACTERIZAÇÃO DA SUPERFÍCIE}

Após a esterilização foi feita a técnica de determinação do ângulo de contato estático ou técnica da gota séssil para cada uma das amostras. As amostras foram colocadas em uma superfície plana, de um goniômetro (gotamarc) (figura 3), utilizando micropipeta de volume $20 \mu 1$, digital, posicionada de forma perpendicular à superfície, funcionando com uma solução de água bidestilada, depositando muito próxima a superfície das placas. De forma padronizar os teste e por serem gotas muito pequenas, foram realizado acompanhamento da mudança de ângulo por 01 segundo e 60 segundos e posteriormente foram analisadas em um software de imagem. $\mathrm{Na}$ análise das fases superficiais foi utilizado um difratômetro de raios-X, Shimadzu modelo XRD-6000. As amostras foram analisadas com ângulos de varredura entre 30 e $80^{\circ}$, passos de $0,002^{0}$ e tempo de $3 \mathrm{~s}$ por passo numa velocidade de $1 \% \mathrm{~min}$. Para análise da rugosidade foi mensurado o parâmetro $\mathrm{Ra}$, utilizando um rugosímetro modelo SURTRATONIC 3, Robson Taylor com cut-off igual a 0.25. As medidas foram tomadas em três direções diferentes, em ângulos de aproximadamente $120^{\circ}$ com dispersão nestas em três direções diferentes. 


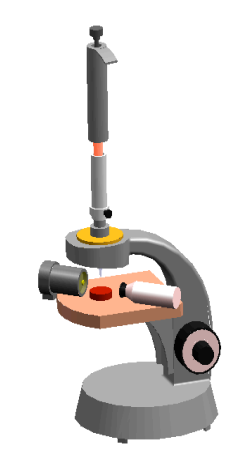

Figura 3 - Aparelho gotamarc, utilizado para medir o espalhamento da gota sobre as amostras.

\section{RESULTADOS E DISCUSSÕES}

A análise da molhabilidade é um fator importante para definir a biocompatibilidade e tem importância primordial na osseointegração dos implantes orais (ALTANKOV G., GROTH T., 1994 \& KASEMO B., 1998, 2002). Ângulos de contatos baixos, significam maior molhabilidade e conseqüentemente melhor avidez da superfície para interagir com o meio que está inserido. Na figura 4, são apresentados os valores dos ângulos de contato para todas as amostras estudadas. Verifica-se que todas as condições estudadas, com exceção apenas da amostra tratada em plasma de hidrogênio por $20 \mathrm{~min}$., apresentaram valores inferiores à amostra não tratada. A melhor condição de molhabilidade foi à mistura dos gases em partes iguais por 20 minutos representado uma diferença de aproximadamente $90 \%$ relação à amostra sem tratamento.

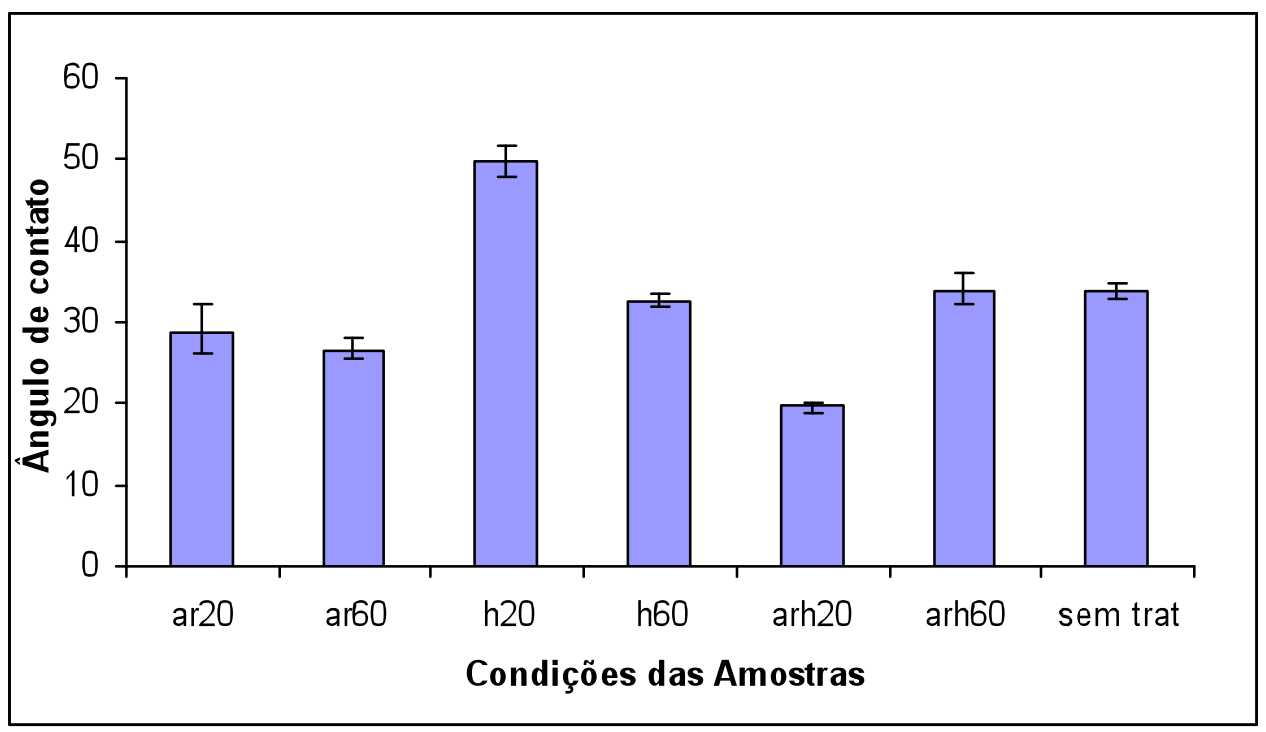

Figura 4 - Gráfico do Ângulo de Contato. 
Analisando os resultados nos ensaios de rugosidade observou-se que, de um modo geral, amostras submetidas ao tratamento de superfície, apresentaram maior rugosidade que àquela não tratada (Figura 5). Observou-se que a relação entre os resultados da rugosidade obtida e os resultados da molhabilidade não estão diretamente relacionados. Isto reforça o fato de que mesmo se a tensão superficial for a mesma, os efeitos de capilaridade de uma superfície sobre um líquido não dependerá diretamente da sua rugosidade. Ou seja, melhor que a rugosidade será analisar o tamanho crítico das micro-cavidades de modo a otimizar o efeito capilar.

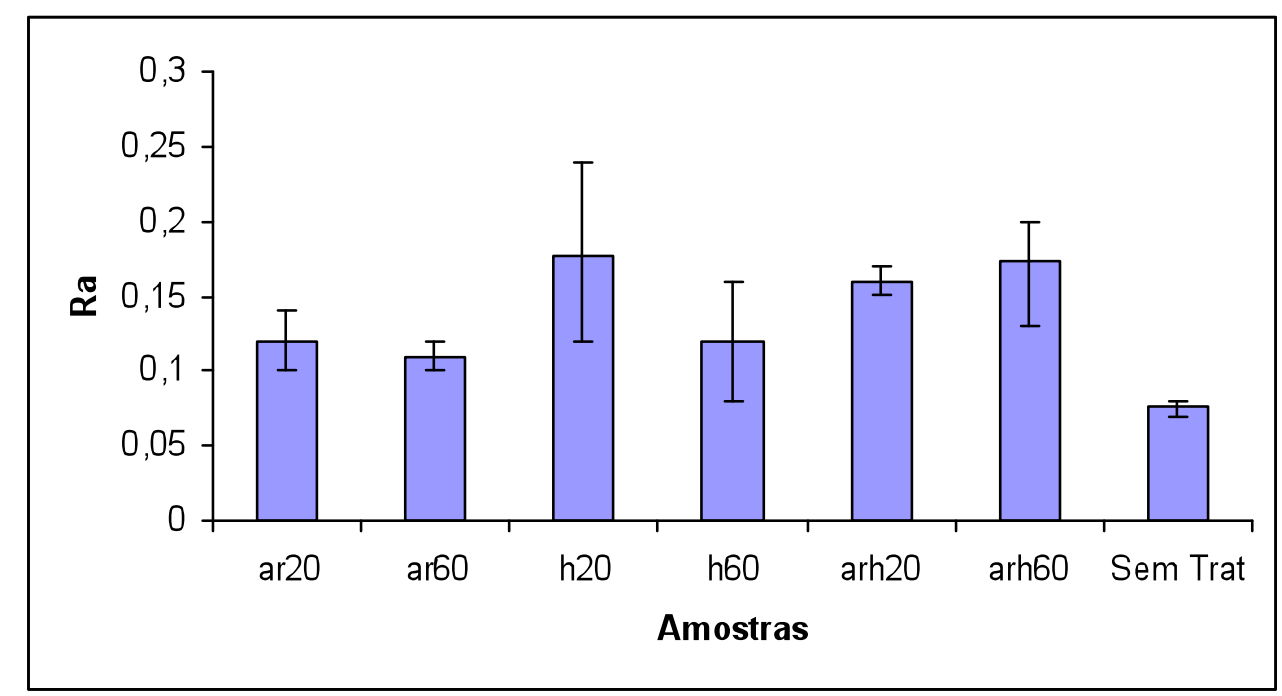

Figura 5 - Medidas e Rugosidade em Ra.

Na figura 6, são apresentados os espectros de difração de raios-X das amostras tratadas. $\mathrm{Na}$ condição de tratamento com argônio durante 20 minutos foi observado uma pobre formação de óxidos, mostrando que nesta condição não foi capaz de promover uma despassivação eficiente da superfície permanecendo maior quantidade de Ti. Na condição onde foi realizada a limpeza prévia com argônio, durante 60 minutos e hidrogênio durante 20 e 60 minutos, aparecem picos mais intensos referentes às fases $\mathrm{TiO}_{2}$ e $\mathrm{Ti}_{3} \mathrm{O}$ e conseqüentemente uma redução na fase de Ti, mostrando que para estas condições, um filme mais espesso dessas fases existe na superfície. Isto acontece pela efetividade da remoção do óxido original (filme passivo) e posteriormente maior reoxidação que as demais amostras. Observando assim que o tratamento usando somente o argônio como gás de limpeza nas amostras de Ti é influenciado pelo tempo do processo. Porém, quando utilizamos somente o hidrogênio, a diferença nas intensidades desses picos para os dois tempos de limpeza utilizados (20 e $60 \mathrm{~min}$.) foi pouco significativa, ou seja, o plasma de hidrogênio foi efetivo até para tempos de limpeza tão pequenos quanto $20 \mathrm{~min}$. 


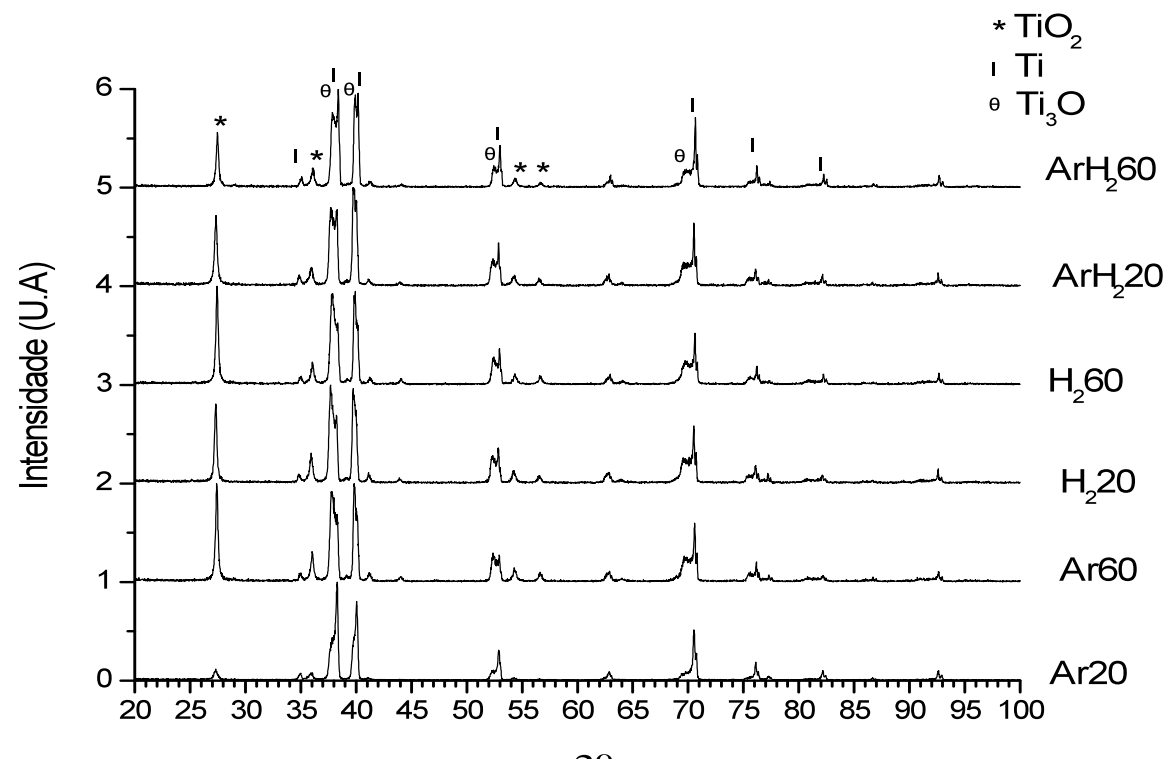

$2 \theta$

Figura 6 - Difração de Raio-X.

\section{CONCLUSÃO}

O presente estudo aponta que a utilização de $\mathrm{Ar}$ e $\mathrm{H}_{2}$ e $\mathrm{Ar} / \mathrm{H}_{2}$ são efetivos para limpeza prévia em Ti na configuração catodo oco e que a rugosidade foi maior em todas as condições tratadas. A melhor condição de molhabilidade foi à mistura dos gases em partes iguais por 20 minutos representado uma diferença de aproximadamente $90 \%$ com relação à amostra sem tratamento, porém não foi observada relação entre a rugosidade e molhabilidade. $\mathrm{Na}$ limpeza com argônio durante 20 minutos, houve pouca formação de óxidos após o tratamento, foi observado também que a limpeza com hidrogênio se mostrou muito eficaz na incorporação de óxidos após o processo de oxidação a plasma. Mais estudos na caracterização da superfície com relação as propriedades mecânicas se torna necessário para investigar a influência do hidrogênio no material aqui estudado.

\section{REFERÊNCIAS}

1. ALBREKTSSON, T., SENNEBY, L. Direct bone anchorage of oral implants: clinical and experimental considerations of the concept of osseointegration. Int. J. Prosthodont, v. 3, n. 1, p.30-41, 1990.

2. ALVES JR. C. Nitretação em plasma pulsado: equipamento, preparação e caracterização de camadas nitretadas. São Paulo, 1995. Tese de doutoradoUniversidade de São Carlos (UFSCar), 1995.

3. ALTANKOV G., GROTH T. Standard recommended practice for surface preparation and marking of metallIc surgical implants. AMERICAN SOCIETY FOR TESTING 
AND MATERIALS. J. Mater. Sci. Mater. Med. 732, 1994. Philadelphia, ATMS, 1990. (ASTM Standard F86).

4. CHANG H.Y. CHANG H.J. YONG H.L, WOOK K.J, GEUN O. Surface Oxide that cleans for a Surface of plasma of atmospheric pressure and Layers . Materials Science and Engineering, 177 -178 711-715.

5. GOMBOTZ W.R, HOFFMAN A.S. Crit. Rev. Biocompat, 4-49, 1987.

6. HSIEH J.H., C. LI Effects of hollow cathode and $\mathrm{Ar} / \mathrm{H}_{2}$ ratio on plasma cleaning of $\mathrm{Cu}$ leadframe Surface and technology. 101-103, 2006.

7. LIU X., CHU P. K., DING C., Surface modification of titanium, titanium alloys, and related materials for biomedical applications. Materials Science and Engineering, 01 69, 2005.

8. KASEMO B. Surface Science 500 656, 2002.

9. KASEMO B, J.LAUSMAA, J. Biomed. Mater. Rev. 22 145, 1988.

10. MITTAL K.L. Surface Contamination: Genesis, Detection and control, vol, prenum Press, New York, 3-46, 1979. 